\title{
Ungleiche Teilhabe - Überlegungen zur Normativität des Medienkompetenzbegriffs
}

\author{
Nadia Kutscher
}

\begin{abstract}
Anhand von Ergebnissen aus eigenen und anderen empirischen Studien zur Internetnutzung Jugendlicher wird die Bedeutung der Habitustheorie von Bourdieu für die Erklärung von Mediennutzung und Aneignungsweisen in der Mediensozialisation expliziert. Vor diesem Hintergrund erfolgt eine Auseinandersetzung mit dem Begriff der ‘Medienkompetenz〉 unter Aspekten milieuspezifischer Medienaneignung und damit verbunden bestimmte (medien-)pädagogische Denkmuster und Handlungsformen als Faktor sozialer Benachteiligung.
\end{abstract}

\section{Ungleichheiten in der Mediennutzung von Jugendlichen}

Neuen Medien wie dem Internet werden im öffentlichen Diskurs vielfach Potentiale für gesellschaftliche und bildungsbezogene Teilhabe zugeschrieben. Die Mediennutzung ist allerdings in sozialstrukturelle Gegebenheiten eingebettet, die neben dem Zugang vor allem die Art und Weise der Nutzung beeinflussen. Hierbei wird das Interdependenzverhältnis von Habitus und Feld relevant und entsprechend kann der Umgang mit Medien je nach Ressourcenverfügbarkeit in Hinsicht auf soziale Teilhabe befördernd oder behindernd wirken.

Eine Reihe empirischer Studien zeigen, dass bezüglich des Zugangs, vor allem aber der Nutzung des Internet soziale Ungleichheiten wirkmächtig sind.

Die JIM-Studie 2008 zählt 97\% der 12- bis 19-jährigen Jugendlichen zu den Online-Erfahrenen (vgl. Feierabend/Rathgeb 2008, S. 46). Betrachtet man diese Zahl aufgeschlüsselt nach Bildungsgruppen zeigt sich, dass Hauptschüler/innen zu 92\% und GymnasiastInnen zu 98\% das Internet nutzen (vgl. ebd.). Dies deckt sich mit den Ergebnissen des (N)Onliner-Atlas 2008, der ebenfalls eine kontinuierliche Verringerung bildungsbezogener Differenzen im Hinblick auf den Internetzugang feststellt ((N)Onliner 2008, S. 15). Allerdings bestehen weiterhin Ungleichheiten in der Medienausstattung: Hauptschüler/innen besitzen etwas seltener einen eigenen Computer bzw. einen eigenen Internetzugang als GymnasiastInnen (Computer: $66 \%$ zu 72\% und Internet: 47\% zu 52\%; vgl. Feierabend/Rathgeb 2008, S. 11). In der Freizeitbeschäftigung mit Medien unterscheiden sich Jugendliche ebenfalls nach Bildungsgruppen. So nutzen Jugendliche mit formal höherer Bildung deutlich öfter Computer und Internet (vgl. Feierabend/Rathgeb 2008, S. 14). 
Während sich die Frage des Zugangs im Laufe der letzten Jahre nachhaltig entdramatisiert zu haben scheint, sind weiterhin deutliche Unterschiede in der Nutzung festzustellen. Prinzipiell entfaltet das Internet seine Potentiale als sogenanntes «Pull-Medium»: Die Angebote des Internet realisieren sich erst durch die Auswahlentscheidungen der Nutzer/innen als Angebotsarrangement (vgl. Iske et al. 2007, S. 66f.). Diese Auswahl erfolgt vor dem Hintergrund der eigenen Interessen, Fähigkeiten und Möglichkeiten sowie auf der Grundlage dessen, was den Nutzer/ innen als Angebot im Internet bekannt ist bzw. von ihnen wahrgenommen wird. In diesem Zusammenhang bilden sich habitualisierte Nutzungspraktiken heraus, die in einem jeweils ungleichen realisierten Nutzungsspektrum resultieren.

In Hinblick auf die informationsbezogene Nutzung des Internets verweisen sowohl die JIM-Studie 2008 als auch die Studien des Kompetenzzentrums Informelle Bildung ${ }^{1}$ Unterschiede je nach Bildungshintergrund der Jugendlichen, insbesondere dahingehend, dass Jugendliche mit formal niedrigen Bildungshintergrund das Internet seltener für Recherche und Information einsetzen als es beispielsweise Gymnasiasten tun (vgl. Feierabend/Rathgeb 2007, S. 48; Iske et al. 2007, S. 78). Diese Ergebnisse werden auch durch Schorb et al. im Rahmen des Medienkonvergenz-Reports bestätigt (vgl. Schorb et al. 2008, S. 16).

Ursache für diese Differenzen sind unterschiedliche Motive in der Nutzung. Jugendliche mit formal niedrigem Bildungsgrad geben beispielsweise als Motive häufig «Zeit vertreiben», «Spass haben» etc. an (vgl. Iske et al. 2004b, S. 7). Sie bevorzugen eher unterhaltungsorientierte Angebote wie z. B. Chatten oder Online-Spiele und auch bei der Informationssuche überwiegen Themen aus dem Unterhaltungsbzw. Fernsehsektor wie z. B. Informationen zu Lieblingsserien, Lieblingsstars etc. (vgl. Otto et al. 2004, S. 14 und 23). Die Gründe für diese Orientierung liegen allerdings nicht in individuellen Entscheidungen, sondern in sozialen und strukturellen lebensweltlichen Erfahrungen der Jugendlichen, wie später noch ausgeführt wird. Bernd Schorb et al. kommen hierbei zu dem Ergebnis, dass Jugendliche mit einem niedrigen formalen Bildungshintergrund häufiger als Jugendliche mit formal hoher Bildung Medien nutzen, um sich in dabei mit ihren Musik, Spiel-, Film- und Fernsehpräferenzen zu beschäftigen (vgl. Schorb et al. 2008, S. 49). Jugendliche mit formal höherem Bildungsgrad praktizieren dagegen eine stärker informationsbezogene Internetnutzung und suchen dabei eher nach politischen Informationen, Nachrichten oder auch nach Informationen für Hausaufgaben (vgl. Otto et al. 2004, S. 23). Auch die Nutzung von Suchmaschinen zur gezielten Informationssuche zeigt eine deutliche Korrelation mit dem formalen Bildungshintergrund (vgl. Iske et al. 2007, S. 78).

Dies kann dahingehend interpretiert werden, dass Gymnasiastlnnen aufgrund der anzunehmenden Nähe ihrer Herkunftsmilieus zur «legitimen Kultur» und ihrer ha-

zusammen mit Hans-Uwe Otto, Alexandra Klein und Stefan Iske 
bituellen Orientierung (Bourdieu) die Suche nach Informationen eher als relevant einschätzen. Darüber liegt die Vermutung nahe, dass die Gründe für eine unterhaltungsorientierte Nutzung auf Seiten der Jugendlichem mit formal niedrigerem Bildungshintergrund auch in der Verweigerung von Anerkennung ihrer Lebenswelt durch die Gesellschaft und die dadurch bedingte Tendenz, sich alternativ Entlastung zu verschaffen, liegen könnten (vgl. Welling 2008). Sofern habituelle Praktiken und Lebensentwürfe gesellschaftlich abgewertet werden und Teilhabeperspektiven als prinzipiell benachteiligend erfahren werden (s. a. World Vision 2007), erscheint es konsequent, dass auch der Bereich der Medien in der Folge zum Feld der Distinktion wird.

Auch in Bezug auf die Einschätzung des Gebrauchswertes der im Internet gefundenen Informationen zeigen sich bildungsbezogene Differenzen: Jugendliche mit hohem formalen Bildungsgrad geben häufiger an, im Internet neue, nützliche und «exklusive», d. h. nur im Internet verfügbare Informationen zu finden als Jugendliche mit formal niedrigem Bildungsgrad (vgl. Iske et al. 2004a, S. 17). Hier liegt die Interpretation nahe, dass ein höherer formaler Bildungsgrad in Verbindung mit weiteren sozialen und kulturellen Ressourcen (Bourdieu) das Erschliessen neuer, vor dem Hintergrund sozial kontextualisierter Anerkennungshierarchien als weiterführend geltender Informationen positiv beeinflussen kann und dass Jugendliche mit formal niedrigem Bildungshintergrund in dieser Hinsicht benachteiligt sind (vgl. ebd., S. 17).

Auch die Registrierung an und die Nutzung von Internetangeboten, die eine Email-Adresse voraussetzen bzw. eine regelmässige Email-Nutzung erfordern (z. B. Newsletter, Messenger-Dienste oder Communitys) stellt häufig eine implizite Hürde dar.

Darüber hinaus tragen in der Nutzung kommunikativer Dienste und Angebote Strategien der «symbolischen Repräsentation des sozialen Status» (Iske et al. 2004b, S. 6) zu sozialer Schliessung bei. In der Nutzung von virtuellen Kommunikationsangeboten, in die eine Vielzahl von Teilnehmer/innen involviert sind, spielen symbolische Repräsentationen von Zugehörigkeit und Identitätsverbalisierungen eine grosse Rolle (vgl. Klein 2008, Tillmann 2008). Diese Formen der kommunikativen Repräsentation von Gruppenzugehörigkeit lassen sich auch als Formen der Distinktion begreifen: Der soziale Status bzw. die soziale oder ethnische Herkunft wird benannt oder durch gezielte Wahl von Chaträumen zum Ausdruck gebracht (vgl. Schäfer/Lojewski 2007, S. 121). Verschiedene Autorlnnen verweisen ebenfalls auf weitere Distinktionsformen im Rahmen der Online-Kommunikation, die sich sowohl auf inhaltlicher Ebene als auch durch kommunikative Formen vollziehen (vgl. Gebhardt 2001, S.9; Henke et al. 2008, S. 474). Soziale Grenzüberschreitungen finden im Internet kaum statt und somit stellen virtuelle Kommunikationsräume vielfach «exklusive Gemeinschaften» dar (vgl. Schönberger 2000, S.35ff; Klein 2008, S. 145ff.; Tillmann 2008). 
Die Autorlnnen des Medienkonvergenz-Reports kommen zu dem Ergebnis, dass Jugendliche mit formal niedrigem Bildungshintergrund bei Web 2.0 Aktivitäten, die sich auf die Bereiche Musik, Foto-Uploads und Videos beziehen, besonders beteiligt sind (vgl. Schorb et al. 2008, Kiessling 2008, S. 21). Dies bestätigen die Befunde der JFF-Studie sowie der JIM-Studie 2008 ebenfalls (vgl. Eggert et al. 2008, S. 152 und 167; Brüggen/Wagner 2008, S.229; Wagner 2008, S. 215; Feierabend/ Rathgeb 2008, S. 48).

Die Daten des Medienkonvergenz-Reports und des JFF scheinen dafür zu sprechen, dass trotz unterschiedlicher Nutzung der Zugang zu gestaltenden Medienpraxen im Internet kein ausschliessliches Privileg formal höher gebildeter Nutzer/ innen ist. Dennoch wird mit diesen Befunden deutlich, inwiefern jugendliche Internetaktivitäten auch durch die Einbettung in lebensweltlich relevante Orientierungs- und Verwertungsmuster vorstrukturiert sind und sich somit auch die Repräsentanz verschiedener Gruppen im Netz auf formaler und inhaltlicher Ebene ausdifferenziert.

Das vielfach euphorisch als konsequente Realisierung von Demokratie gefeierte Web 2.0 bedarf in diesem Zusammenhang weitergehender Analysen. Die präsentative Nutzung beispielsweise in Form von Foto-Uploads ist jedoch in Hinblick auf die Realisierung von Teilhabe deutlich zu unterscheiden von einer aktiv-gestaltenden Mitgliedschaft in Communitys, die Interessen organisier- und durchsetzbar macht oder einer mit materiellem Einsatz und Software-Kenntnissen verbundenen Beteiligung in «Second Life».

Fred Schell stellt in dieser Hinsicht fest, dass die technische Möglichkeit der aktiven Nutzung und (gesellschaftlichen) Partizipation nicht auch zwingend zu einer entsprechenden Nutzung führt, sondern dass dies jeweils spezifische Medienkompetenz voraussetzt (vgl. Schell 2008, S. 10).

Vor dem Hintergrund unterschiedlicher Nutzungsweisen und der damit verbundenen ausdifferenzierten Verortung im Internet liegt darüber hinaus die Interpretation einer Ausdifferenzierung von Räumen im Netz nahe (vgl. Kutscher 2009).

\section{Medienhandeln, Habitus und Feld}

In den dargestellten Ergebnissen wird somit deutlich, dass aufgrund unterschiedlicher Präferenzen und Fähigkeiten die Nutzung von Angeboten im Internet verschieden verteilt ist und damit gleichzeitig eine Ausdifferenzierung von Nutzer/ innengruppen innerhalb unterschiedlicher «Orte» im Netz erfolgt.

Vermeintlich subjektive Nutzungspräferenzen lassen sich allerdings erst in Zusammenhang mit dem soziokulturellen Hintergrund der jugendlichen Internetnutzer/ innen sinnvoll einordnen. In diesem Zusammenhang erweisen sich sowohl Erfahrungen ausserhalb des Internet wie innerhalb des Internet als bedeutsam. Die materiellen, kulturellen und sozialen Ressourcen, die Nutzer/innen ausserhalb des Internet zur Verfügung stehen (z. B. im lebensweltlichen Kontext erworbene Kom- 
petenzen im Umgang mit verschiedenen Medien sowie Interessens- und Präferenzmuster, soziale Unterstützung bei der Mediennutzung, Peer-Strukturen sowie technische Ressourcen) erweisen sich hierbei als ebenso relevant wie die Erfahrungen die Jugendliche innerhalb des Internet als Feld machen. Hierbei erscheinen sowohl die medialen Nutzungsanforderungen bestimmter Internetangebote, die inhaltliche Ausrichtung der Angebote wie auch die sozialen Dynamiken innerhalb verschiedener Angebote bedeutsam und weisen auf strukturelle Aspekte hin, die über die Medienkompetenz der Nutzer/innen selbst hinaus gehen (vgl. Iske et al. 2007, S. 69; Klein 2008, Tillmann 2008).

So zeigt sich unter anderem, dass Nutzungsweisen mit den kulturellen Ressourcen (hier verdeutlicht anhand des Indikators formale Bildung) zusammenhängen und spezifische Nutzungsweisen wiederum miteinander korrelieren. Dies kann erklärt werden dadurch, dass Handeln in Anerkennungsstrukturen und lebensweltliche Relevanzen eingebettet und jeweils sozial sinnhaft ist, d. h., je nach lebensweltlichem Kontext sind unterschiedliche Handlungsprioritäten relevant (vgl. Bittlingmayer/Bauer 2006, S. 215). So kann beispielsweise bei dem Nutzungsmotiv Zeitvertreib die Bedeutung seriöser Informationen sekundär sein ${ }^{2}$ während bei einer saufstiegsorientierten> Nutzung die Fokussierung auf gesellschaftlich anerkannte Praxen in den Vordergrund rückt. Innerhalb eines lebensweltlichen Zusammenhangs können also Nutzungspräferenzen entsprechend dem jeweiligen sozialen Kontext und der damit verbundenen Alltagsrelevanzen als sinnhaft interpretiert werden. Das bedeutet allerdings nicht, dass von «objektiven» Lebensbedingungen ein bestimmtes «subjektives» Denken und Handeln kausal abgeleitet werden kann, sondern letzteres das Ergebnis eines komplexen Zusammenspiels von Habitus, Feld und Raum (Bourdieu) ist, das u. a. daran deutlich wird, dass die Nutzungspraxen zwischen den formalen Bildungsgraden nicht völlig unterschiedlich, sondern fluide Übergänge und Ausnahmen beobachtbar sind Internetnutzer/innen bewegen sich mit unterschiedlichen Motiven, unterschiedlichen Voraussetzungen und verorten sich in verschiedenen sozialen Netzwerken im virtuellen Raum. In diesem Zusammenhang spielen verschiedene Distinktionsweisen eine Rolle, die sich innerhalb der kommunikativen Prozesse im Netz ausdrücken. Alexandra Klein hat dies beispielhaft an einem Beratungsforum verdeutlicht, in dem sich beispielsweise Themen von Hauptschüler/innen innerhalb einer primär durch Realschüler/innen und GymnasiastInnen dominierten Nutzer/innenschaft nicht etablieren konnten (vgl. Klein 2005, S. 15f.).

Adressiert also ein Internetangebot implizit oder explizit (über Inhalte, Angebotsformen o. Ä.) eine spezifische Nutzer/innenschaft, so kann dies dazu führen, dass

2 Wobei beispielsweise das Motiv Zeitvertreib unterschiedliche Ursachen haben kann: Die Erfahrung von Chancenlosigkeit bei der Suche nach einem Ausbildungs- oder Arbeitsplatz bildet einen anderen Hintergrund dafür als das Ziel, sich an einem langweiligen Tag einmal zwischendurch abzulenken. 
das Angebot faktisch nicht mehr für alle offen ist und ein "Voice Divide» (Klein 2004) entsteht. In der Konsequenz führt die Erfahrung, mit den eigenen Interessen nicht gehört zu werden, dazu, dass bestimmte Nutzer/innengruppen Internetseiten dominieren und auf diese Weise durch kommunikative Prozesse sowie begünstigt durch Angebotsstrukturen und -inhalte eine Homogenisierung von Räumen im Netz stattfindet. Somit sind es informelle Prozesse und Praxen, die Räumlichkeit strukturieren. Auch im Netz bedingen also soziale Strukturen Handlungen und Handlungen aktualisieren soziale Strukturen (vgl. Löw 2001).

In diesem Zusammenhang haben mediale, inhaltliche und personale Passungsverhältnisse (vgl. Klein 2004) für das Zusammenspiel zwischen Nutzung und Angebot hohe Bedeutung, d.h., innerhalb dieser drei Dimensionen entscheidet sich die Frage, inwiefern das jeweilige Internetangebot einen Ort für die betreffenden Nutzer/innen darstellt. Wenn nun ein Internetangebot eine bestimmte Nutzungsweise oder bestimmte Inhalte vornehmlich bedient und ermöglicht, so werden implizit diejenigen, die andere Nutzungsweisen oder Inhalte bevorzugen bzw. alltagskontext- oder ressourcenbedingt andere Nutzungsgewohnheiten haben, exkludiert. Auf der Grundlage dieser Überlegungen sowie der Bedeutung sozialer und kultureller Ressourcen innerhalb kommunikativer Prozesse und Räume wird deutlich, dass die dargestellten empirischen Ergebnisse kein Resultat zufälliger individueller Präferenzen sind, sondern das Ergebnis komplexer aber dennoch ressourcenabhängiger relativer Positionierungen im sozialen Raum, die gleichzeitig im virtuellen Raum so etwas wie Orte konstitutieren, die mit Bourdieu als Felder oder mit Harvey und Lefebvre als relativer und relationaler Raum bzw. Raumrepräsentationen bezeichnet werden können. So stellen auch Internetseiten Felder dar, innerhalb derer Machtverhältnisse verhandelt werden. Betrachtet man das Verhältnis von Habitus und Feld, so wird deutlich, dass ungleiche Mobilitätsoptionen für die einzelnen Akteure je nach Habitus und Kapitalausstattung bestehen. So kann an vielen Internetseiten, die Jugendliche quer durch alle Schichten erreichen wollen, nachvollzogen werden, dass sich dort jedoch häufig vor allem formal höher gebildete Jugendliche wieder finden und andere Gruppen sich darin nicht (mehr) etablieren (können). Beispiele hierfür sind besonders textbasierte an Jugendliche gerichtete Seiten, innerhalb derer sich vor allem formal höher gebildete Nutzer/ innengruppen etablieren oder Chaträume, innerhalb derer u. a. stereotype Kommunikationen der Beziehungsanbahnung stattfinden und die - soweit nachvollziehbar - eher formal niedriger gebildete Nutzer/innen binden.

Dies hat wiederum weitgehende Implikationen für die Frage, wessen Interessen im Internet Gehör finden bzw. durchsetzungsfähig sind (vgl. Kutscher 2007, S. 188) bzw. auch dafür, wie Ausdifferenzierungen von Mitgliedschaften und auch Schliessungsprozesse in Communitys erfolgen.

Vor dem Hintergrund der dargestellten Ungleichheiten in der Mediennutzung und ihrer impliziten Bedeutung für soziale Teilhabe - stellt sich die Frage, inwie- 
fern die Vermittlung von Medienkompetenz das Ungleichheitsproblem verringern helfen könnte.

\section{Medienkompetenzbegriffe}

Nach Baacke geht es bei der Aneignung von Medienkompetenz um den Erwerb spezifischer kognitiver Strukturen, die dazu befähigen, «in die Welt aktiv aneignender Weise auch alle Arten von Medien für das Kommunikations- und Handlungsrepertoire von Menschen einzusetzen» (Baacke 1996, S. 119, Hervorhebung im Original). Das Baackesche Medienkompetenzmodell beinhaltet in seinen Dimensionen verschiedene Aspekte, die unterschiedlich stark in der medienpädagogischen Forschung und Praxis thematisiert werden. Hierbei ist ein Aspekt, der häufig wenig reflektiert wird, die Frage sozialer Ungleichheiten im Kontext der Mediennutzung - obwohl er in Dieter Baackes Begriff der Medienkritik deutlich als kritisch-reflexive Auseinandersetzung mit gesellschaftlichen Machtstrukturen eine Rolle spielt.

Unterschiede in der Mediennutzung werden im medienpädagogischen Kontext vielfach aus einer individualisierenden oder kulturalisierenden Perspektive thematisiert. Häufig werden dabei sozialstrukturelle Bedingungsfaktoren für Aneignung und Teilhabe und die Bedeutung lebensweltlicher Relevanzen bestenfalls problematisierend erwähnt und bestimmte Mediennutzungsformen a priori als defizitär diskreditiert und abgewertet. Dass viele Jugendliche vorrangig «nur» chatten und wenig informationsorientiert z. B. Wikipedia (das hinsichtlich seiner Qualität auch kritisch zu hinterfragen ist) oder andere primär informationsbezogene Seiten nutzen - und, wie in verschiedenen Studien gezeigt wurde, es sich hierbei vielfach (aber nicht ausschliesslich) um Jugendliche mit formal niedrigerem Bildungshintergrund handelt (vgl. exemplarisch Wagner 2008, Iske et al. 2007 und 2008) - ist empirisch dokumentiert. Allerdings bedarf es differenzierter Analysen bezüglich der Frage, welche Ursachen dem zugrunde liegen und es stellt sich die Frage, ob eine derartig unterschiedliche Mediennutzung Resultat der Ausbildung einer medialen «Unterschichtskultur» ist, die sich reproduziert oder diesen Unterschieden differenziertere Ursachen zugrunde liegen.

Vor diesem Hintergrund drängt sich geradezu die Frage nach dem normativen Gehalt des Medienkompetenzbegriffs und eine Reflexion auf seine Verwendung auf, da letztere - so ist zu vermuten - vielfach zur Reproduktion sozialer Ungleichheiten beiträgt und selbst Gegenstand eine kritischen, machttheoretischen Reflexion sein müsste.

Miriam Schäfer und Johanna Lojewski haben unterschiedliche Interpretationen des Medienkompetenzbegriffs herausgearbeitet. Dabei wird deutlich, dass jeweils Aspekte von Wissen, Reflexion und Handeln darin vorkommen, sie aber jeweils unterschiedliche implizite normative Konnotationen aufweisen. 
Das Konzept von Baacke (1998) stellt laut Lojewski/Schäfer das am meisten rezipierte Modell in der Medienpädagogik dar und bildet die Basis für verschiedene andere Konzepte von Medienkompetenz. Darin ist besonders die Einbettung in das Konzept der kommunikativen Kompetenz hervorzuheben sowie die Annahme, dass jeder Mensch bereits über diese Kompetenz verfügt, sie aber dennoch gelernt, geübt und weiterentwickelt werden muss. Der Erwerb von Medienkompetenz findet laut Baacke nicht nur in der Schule, sondern in allen lebensweltlichen Kontexten statt. Dabei berücksichtigt Baacke nicht nur die Ebene der Vermittlung, sondern auch die Ebene des Medienhandelns. Durch die beiden Dimensionen Medienkritik und Medienkunde wird ein kognitiv ausgeprägter Medienkompetenzcharakter betont (vgl. Schäfer/Lojewski 2007, S. 67). Baacke problematisiert allerdings später die rationalistische Verengung seines Konzeptes in der medienpädagogischen Rezeption und Diskussion (vgl. Welling 2005, S. 4).

Gerhard Tulodziecki (1997) entwickelt auf der Grundlage des Baackeschen Modells das Konzept einer handlungs- und interaktionsorientierten Medienerziehung und -bildung für den schulischen Kontext. In ihrer Analyse machen Schäfer/Lojewski die starke Fokussierung der kritisch-reflexiven Dimension von Medienkompetenz in Tulodzieckis Modell und damit den normativen Charakter seines Konzeptes deutlich. Demnach scheint nur eine im qualifikationsorientierten Sinn «bildungsrelevantes Mediennutzung eine gute Mediennutzung zu sein, während eine unterhaltungsorientierte Nutzung als weniger anerkennenswert betrachtet wird. Darüber hinaus wird hierbei der informelle Erwerb von Medienkompetenz vernachlässigt, indem Medienkompetenz fast ausschliesslich als Aufgabe von institutionalisierter Bildung und Erziehung definiert wird (vgl. Schäfer/Lojewski 2007, S. 69).

Verschiedene Autorlnnen hinterfragen die definierten Ziele und Dimensionen der Medienkompetenz bezüglich des Mediengebrauchs im Alltag, der in spezifische soziale und kommunikative Praxen der Lebenswelt ${ }^{3}$ eingebettet ist und von soziokulturellen Ressourcen beeinflusst wird. Hierbei wird insbesondere die Problematik des zweck- und zielgerichteten Mediengebrauchs sowie die Betonung von Qualifizierungsaspekten als legitimer Praxis kritisch diskutiert (vgl. Bader 2001, Bausch/ Sting 2001, Schäfer/Lojewski 2007, Welling 2008, S. 61ff.).

Stefan Welling kritisiert u. a. die häufig zweckrationale Ausrichtung medienpädagogischen Handelns und das damit verbundene Missverständnis, dass Mediennutzer/innen (und hierbei insbesondere auch Jugendlichen) eine kognitiv-rationale Zweck-Mittel-Orientierung zugeschrieben bzw. bei ihnen habituell vorausgesetzt wird (vgl. Welling 2008, S.46). In Auseinandersetzung mit Konzepten medienpädagogischer Professionalität von Kai-Uwe Hugger zeigt er auf, dass unterschiedlichen Perspektiven ${ }^{4}$ medienpädagogischer Arbeit mit Jugendlichen hierbei prä-

3 Vgl. hierzu auch die Ergebnisse der JFF-Studie 2008 (Brüggen/Wagner 2008, 225)

4 beschützend-wertvermittelnd/kulturkritisch-geisteswissenschaftlich, technologisch-funktional bzw. bildungstechnologisch, gesellschaftskritisch-wissenschaftszentriert und handlungsorientiert 
gend wirken und macht dabei deutlich, wie diese jeweils unterschiedliche Foki bezüglich dessen, was angeeignet bzw. als Motiv und Interesse vorausgesetzt wird, darstellen und je nachdem eingeschränkt für offene Jugendarbeit und ihre spezifischen Zielgruppen geeignet sind (vgl. Welling 2008, S. 56ff.).

Während in den Modellen von Baacke und Tulodziecki Ziele von Medienkompetenz als spezifisch kompetenter Gebrauch von Medien definiert werden, fokussiert Norbert Groeben statt einer Zieldimension die Prozessdimension der Mediennutzung und verweist damit auf eine nutzer/innenorientierte Perspektive von Medienkompetenz. Auf diese Weise kann eine Hierarchisierung und Gewichtung der einzelnen Dimensionen und die damit verbundene normative Setzung vermieden werden (vgl. Groeben 2002, S. 162f).

Schäfer/Lojewski weisen darauf hin, dass Groeben die Dimensionen von Medienkompetenzen im Medienrezeptionsprozess selbst verortet, der potentielle Anlässe zur Entwicklung von Medienkompetenz bietet. Diesbezüglich seien hervorzuhebende Merkmale von Groebens Modell neben der Prozessperspektive vor allem die Dimensionen der medienbezogenen Genussfähigkeit und der Anschlusskommunikation. In Bezug auf medienbezogene Genussfähigkeit kritisiert Groeben, dass diese lange Zeit nicht ausreichend aufgearbeitet worden ist, zum Einen, weil die Ausdifferenzierung und Förderung von Kritikfähigkeit immer oberste Priorität besessen hat, und zum Anderen, weil rein unterhaltungsmotivierte Rezeptionsweisen zumeist abgewertet werden (vgl. Groeben 2002, S. 164). Durch die Betonung der Berechtigung einer unterhaltungsmotivierten Mediennutzung wird eine differenz- statt defizittheoretische Betrachtungsweise von Mediennutzungspraxen möglich. Anschlusskommunikation als eine strukturelle Voraussetzung für alle oben genannten Dimensionen der Medienkompetenz stellt Groeben zufolge das grundlegende Prinzip bzw. Vorgehen der Mediendidaktik dar und meint hierbei nicht nur die mediendidaktisch intendierte Kommunikation, sondern auch Kommunikationen mit Freunden und Familie im informellen Bereich. Damit wird die Tatsache einbezogen, dass der Erwerb von Medienkompetenz auch bildungs- und milieuabhängig ist (vgl. Groeben 2002, S. 184). Nicht nur der materielle Rahmen, der über die Verfügbarkeit und den Zugang zu Medien entscheidet, sondern auch die sozialen Ressourcen und Netzwerke, also mit wem sich worüber und in welcher Form im Rahmen der Anschlusskommunikation ausgetauscht werden kann, beeinflussen massgeblich die Rezeption und Verarbeitung medialer Angebote.

Durch die Verortung des Erwerbs von Medienkompetenz im Mediennutzungsprozess selbst wird eine subjektzentrierte Sichtweise von Mediennutzung und damit auch von Medienkompetenz möglich, die damit auch jenseits einseitiger normativer Zielorientierungen betrachtet werden kann und hermeneutische Zugänge zu lebensweltlichen Relevanzen ermöglicht.

Allerdings gilt es zu bedenken, dass eine nutzer/innenorientierte Perspektive unter Berücksichtigung unterschiedlicher situationaler und milieuspezifischer Pro- 
blem- und Motivations- bzw. Interessenslagen zwar eine differenz- statt defizittheoretische Sicht auf unterschiedliche Mediennutzungsweisen erlaubt, jedoch auch in sich die Gefahr birgt, die Verantwortung für den Erwerb von Medienkompetenz und für medienvermittelte Bildungsprozesse allein dem Subjekt zuzuschreiben und gesellschaftliche Benachteiligungsstrukturen zu vernachlässigen.

Denn, was als bildungsrelevante Mediennutzung gilt, hängt wiederum damit zusammen, welche gesellschaftliche Vorstellung von «legitimer» Bildung im Sinne Bourdieus vorherrscht und wie diese im Kontext der Mediennutzung interpretiert wird. Das bedeutet, dass sofern Bildungserfolge über gesellschaftlich verwertbaren Informations- und Wissenserwerb definiert werden, auch die Funktion von Medien in Bildungskontexten bewertet wird: Nur die Medien, die potentiell in der Lage sind, institutionell verwertbaren Informations- und Wissenserwerb zu fördern, erhalten den Status der «kulturellen Legitimität» (Schäffer 2000) und dies geht zwangsläufig einher mit einer Unterscheidung zwischen kulturell legitimen und illegitimen Formen der Mediennutzung, d. h., einzelne Medienpraxen gelten als wertvoller als andere (vgl. Schäfer/Lojewski 2007, S. 9 und 92). In der medienpädagogischen Debatte werden diese zugrunde liegenden sozial kontextualisierten und ungleichen Anerkennungsstrukturen häufig als kulturelle bzw. von individuellen Entscheidungen geprägte Präferenzen thematisiert. Eine Kulturalisierung dieser Ungleichheiten scheint jedoch ein problematischer Weg, denn hierbei geraten die Bedingungsstrukturen und Machtverhältnisse, vor deren Hintergrund die Bewertung spezifischer Nutzungsweisen aber auch die Durchsetzungsfähigkeit bestimmter Beteiligungsmöglichkeiten sich figurieren, aus dem Blick.

In diesem Zusammenhang ist ebenfalls die häufig wiederholte These der prinzipiell durch den Migrationshintergrund per se benachteiligten Migrantlnnen ist in diesem Zusammenhang noch einmal zu relativieren: Heinz Bonfadelli und Priska Bucher kommen in einer Studie 2007 zu dem Schluss, "dass die Migrationsjugendlichen als Gruppe genauso wenig wie Schweizer Jugendlich homogen sind und dass neben dem Faktor «Migrationshintergrund die soziale Herkunft und das eigene Bildungsniveau sowie das Alter und Geschlecht ebenfalls einen wichtigen Einfluss auf den Medienumgang haben» (Bonfadelli/Bucher 2007, S.150; vgl. hierzu auch Bonfadelli/Moser 2007). Die Ausdifferenzierung von Nutzung ist also nicht so eindimensional, wie es häufig angesichts quantitativer Ergebnisse zu sein scheint. Es scheint aber Muster zu geben, die deutlich auf soziodemographische Aspekte und hierbei insbesondere auf die Frage von Bildungsvoraussetzungen und sozialem Kapital verweisen.

Das, was als kompetente oder inkompetente Mediennutzung wahrgenommen wird, bewegt sich hier innerhalb von Räumen - oder um mit Bourdieu zu sprechen: innerhalb von Feldern - die durch Machtverhältnisse strukturiert sind. Bettina Pirker spricht dabei unter Bezugnahme auf Massey und Castells von "doing space», innerhalb dessen sich Distinktionsprozesse abspielen (vgl. Pirker 2009). 
Doch diese Distinktion spiegelt sich auch in einem Medienkompetenzbegriff, der sich stark auf eine «bildungsbürgerliche» Konnotation von bildungsbezogenem Handeln bezieht. Konkret spiegelt sich das in bestimmten medienpädagogischen Angebotsformen und Möglichkeiten, die für Mitwirkung vorgesehen sind: Projekte, die produktorientierte, verbalisierende Ausdrucksformen bevorzugen oder auch in der verwendeten Sprache aber auch in Bildungszielen, die bei medienpädagogischen Angeboten formuliert werden (vgl. hierzu auch Niesyto 2000 und 2007). Dabei erfolgt mit dem guten Willen, Teilhabe zu ermöglichen, die implizite Abwertung lebensweltlicher Handlungsbezüge und Nutzungsweisen, die anders sind.

Helmut Bremer weist am Beispiel von Lehr-Lern-Prozessen darauf hin, dass pädagogische Beziehung und Kommunikation durch gesellschaftliche Gestaltungsmuster vorstrukturiert sind. Dabei spannen die Lehrenden ein «Kräftefeld» auf, in dem sich die Schüler/innen «je nach ihrem Habitus spezifisch platzieren dürfen und müssen» (Bremer 2007, S. 273 - Hervorhebung im Original). Über den engen Kontext von Lehr-Lern-Verhältnissen hinaus, so Bremer, kann jedes didaktische Setting einen ermöglichenden oder verhindernden Effekt haben und dies abhängig von den jeweiligen Dispositionen und Habitus der Lernenden und Lehrenden (vgl. ebenda). In der Analyse der Ergebnisse einer empirischen Studie zeigt er, dass in den pädagogischen Konzepten Habitus-Schemata der PädagogInnen einfliessen, die sich dergestalt äussern, dass Lehrer die «akademischen Milieus angehören, in ihrer Arbeit sehr grossen Wert auf der [sic] Stärkung und Förderung von Individualität im Bildungs- und Lernprozess [legen], während von PädagogInnen aus weiter unten stehenden Milieus auch gemeinschaftliche und pragmatische Aspekte betont werden» (Bremer 2007, S. 277). Aufgrund der Habitusschemata komme es somit zu «Fehlwahrnehmungen und Verzerrungen, in deren Folge implizite «Messlatten` von «erwünschtem` Verhalten wie auch von vermuteten Voraussetzungen in die pädagogische Arbeit mit hineingetragen werden» (Bremer 2007, S. 278). Wenn es so ist, dass "pädagogische Kommunikation klassenkulturell geprägt» (Bremer 2007, S. 280) ist - und dafür spricht die Beobachtung der Praxis auch im medienpädagogischen Bereich -, dann stellt sich die Frage, inwiefern auch hier normative Aspekte der Bewertung einer 〈richtigen〉, ‘produktiven〉 Mediennutzung und darauf bezogener Medienbildung bzw. auf der anderen Seite einer 〈falschen〉, 〈unproduktiven` Nutzung, die als zu überwindend thematisiert wird, einer Reflexion im Kontext des Medienkompetenzbegriffs zugänglich gemacht werden können ${ }^{5}$.

5 Die Autorlnnen der JFF-Studie weisen ebenfalls auf die normativen Aspekte des Medienkompetenzbegriffs hin und beschreiben diesbezüglich Benachteiligungen in Bezug auf die Bereiche Teilhabe am sozialen, kulturellen und politischen Leben, selbstbestimmtes Handeln mit den Medien und souveräne Lebensführung mit den Medien (Brüggen 2008, S. 206f.). 


\section{$4 \quad$ Alternative Zugänge zu Mediennutzung und Bildung}

Im Gegensatz zu der Setzung normativer Anforderungen an eine «kompetente» Mediennutzung ermöglicht ein hermeneutischer Zugang und eine Reflexion auf das zugrunde liegende Zusammenspiel von Habitus und Feld ein erweitertes Verständnis der Ermöglichung von Teilhabechancen. In diesem Zusammenhang sei auf die Studie des JFF-Medieninstituts verwiesen, die diesen Zugang wählt. Hierbei wird versucht, auf empirischer Basis einen Medienkompetenzbegriff aus Nutzer/innensicht- insbesondere benachteiligter Jugendlicher - zu rekonstruieren. Als Dimensionen von Kompetenz im Medienumgang arbeiten die Autorlnnen dabei folgende Aspekte heraus (vgl. Brüggen 2008, S. 192-199):

- Wissen über Computer, Handy und Spielkonsole und Bedienfertigkeiten

- Sich mit Hilfe des Internet zu informieren

- Sich mit PC und Internet den Weg in die Berufstätigkeit ebnen

- Kommunikationsmöglichkeiten über Internet und Handy nutzen

- PC und Internet als Produktionsmittel nutzen

- Spielebezogene Kenntnisse

- Wissen aus der «realen Welt», Fremdsprachenkenntnisse und soziale Fähigkeiten einbringen.

Die Autorlnnen der Studie benennen als auffällig, dass in den Äusserungen der Jugendlichen vor allem die soziale Einbettung der Handlungen und Kompetenzen in ihre Lebenswelt eine zentrale Rolle spielt.

Stefan Welling zeigt in seiner empirischen Studie, welche Bedeutung gerade körperlich-aktionistische und kollektive Handlungspraxis im Alltag benachteiligter Jugendlicher hat und dass vielfach die biographischen Orientierungen dieser Jugendlichen mit bestimmten Formen der Computermedienpraxis nicht vereinbar sind. Er gibt damit einen Hinweis für Ansätze in der (auch medien-)pädagogischen Arbeit mit dieser Zielgruppe und macht deutlich, dass gerade Jugendzentren eine «sozialräumliche Gelegenheitsstruktur» für alternative Ansätze sein können. Das in seiner Erhebung beobachtete «episodenhafte Handeln» sozial benachteiligter Jugendlicher auch im Medienkontext interpretiert er als Negation der bzw. Alternativerfahrung zur erlebten Alltagspraxis und als Möglichkeit, Anerkennung zu erlangen, die den Jugendlichen im berufsbiographischen Kontext verwehrt bleibt (vgl. Welling 2008, S. 266-273).

Horst Niesyto (2007) kritisiert die fehlende Erfahrungs- und Lebensweltorientierung in der medienpädagogisch vorherrschenden Praxis und ihren Konzepten. Er bemängelt hierbei vor allem die einseitig kognitiv-planerischen, an Wissenserwerb orientierten Konzepte der Medienbildung, die die sozial-kommunikativen und sozial-ästhetischen Dimensionen von Kompetenzbildung sowie die schicht- und milieuspezifischen Mediennutzungen vernachlässigen (vgl. Niesyto 2007, S. 163ff.). 
Diesen Mängeln setzt Niesyto (2000) ein zielgruppenspezifisches Konzept der Medienbildung entgegen, das an den Relevanzmustern, Orientierungen, Praxen und Kompetenzen von Kindern und Jugendlichen aus benachteiligenden Verhältnissen ansetzt und ihnen in wertschätzenden Arrangements neue Erfahrungen, Kompetenzen und Reflexionsgelegenheiten ermöglicht.

Ähnlich wie Niesyto kritisiert Welling (2005) die Vernachlässigung des spielerischen und affektiv-emotionalen Zugangs der Jugendlichen zu Medien in der medienpädagogischen Praxis sowie die fehlende Passungsfähigkeit zwischen dem ziel- und zweckgerichteten Mediengebrauch in (medien-)pädagogischen Kontexten und den kollektiven, nicht zweckorientierten Praxen der Jugendlichen. Eine zweckrationale Verkürzung der Medienpraxis reflektiert Welling zufolge nicht die milieuund jugendkulturspezifischen Handlungspraxen und biographischen Relevanzen der AdressatInnen und führt dazu, dass sich diese den klassischen Ansätzen handlungsorientierter Medienpädagogik eher entziehen (vgl. Welling 2005, S. 213ff.). Vor diesem Hintergrund stellt gerade das Feld der offenen Jugendarbeit einen etablierten Ort für Jugendliche in benachteiligenden Verhältnissen dar, in dem grundlegende Erfahrungen bezüglich der pädagogischen Sensibilität im Umgang mit dieser Zielgruppe vorliegen, und bietet somit Chancen, eine alternative Idee von Medienpädagogik zu realisieren.

Welling weist darauf hin, dass Professionalität in diesem Kontext vor allem die Kunst darstellt, eine jeweils adressatInnen- und arbeitsadäquate Vorgehensweise zu wählen und aktuelle Tendenzen der Rationalisierung pädagogischer Arbeit, die eine starke Produktorientierung mit sich bringen, kritisch zu reflektieren. Er warnt vor den Folgen der Rationalisierung von Bildung in diesem Feld, die auch mit Ressourcenkürzungen einhergehen und nicht ohne Auswirkungen auf die pädagogischen Ansätze im Feld der medienbezogenen Jugendarbeit bleiben. Dies bedeutet, immer wieder neu sowohl den Jugendlichen und ihren Entwicklungen Raum und Zeit zu geben als auch Input, Struktur und Anregungen durch die Professionellen zu geben und auf diese Weise durch das Schaffen von «Anregungsmilieus» Möglichkeiten und Fähigkeiten erweiternd zu handeln (vgl. Welling 2008).

\section{$5 \quad$ Fazit}

Die dargestellten Überlegungen weisen darauf hin, dass Ungleichheiten in der Mediennutzung in den lebensweltlichen Praxen verankert und in gesellschaftliche Ungleichheitsstrukturen eingebettet sind. In diesem Zusammenhang erscheint ein reflexiver Umgang mit dem Medienkompetenzbegriff, der habituell-normative Aspekte in seiner theoretischen und praktischen Ausdeutung zugänglich und bearbeitbar macht, erforderlich um nicht im medienpädagogischen Kontext Bildungsund Teilhabebenachteiligungen zu reproduzieren. Es geht hierbei darum, Möglichkeiten zu eröffnen, um an dem durch gesellschaftliche Machtverhältnisse und Regeln strukturierten «Spiel» teilzunehmen und damit auch Möglichkeiten zu einer 
Aneignung von gesellschaftlich als relevant erachtetem Wissen und Praxen zu eröffnen. Es bedeutet jedoch auch, Machtverhältnisse und habituelle Differenzen zu reflektieren und vor diesem Hintergrund die fraglose Setzung bestimmter Nutzungsweisen als produktiv, sinnvoll etc. zu hinterfragen und lebensalltagsbezogen unterschiedliche Nutzungsweisen und Ausdrucksformen anzuerkennen.

Ein kritisch-reflexiver Medienkompetenzbegriff und eine darauf begründete Medienbildung müsste somit einerseits darauf zielen, Teilhabe zu ermöglichen, indem Wissen und Kenntnisse erworben werden können, die für gesellschaftliche Teilhabe wichtig sein können (und das auch in einer bildungstheoretischen Thematisierung transparent machen). Darüber hinaus gilt es jedoch, Machtverhältnisse in Vorstellungen von kompetenter Mediennutzung angesichts lebensweltlicher Ungleichheiten aufzudecken und damit die Idee der «richtigen` normativ aufgeladenen und vielfach bildungsbürgerlich konnotierten Idee von Medienkompetenz in Frage zu stellen. Kurz: es geht um die Gleichzeitigkeit einer Ermöglichung von Teilhabe durch die Vermittlung von Fähigkeiten und Möglichkeiten, die sich an einer bildungsbezogenen Weiterentwicklung der Personen orientiert und einer Teilhabe durch Anerkennung, die sich auf eine Veränderung von Strukturen richtet.

\section{Literaturangaben}

Baacke, Dieter. «Medienkompetenz - Begrifflichkeit und sozialer Wandel.» Medienkompetenz als Schlüsselbegriff. Herausgegeben von Antje von Rein. Bad Heilbrunn: Klinkhardt, 1996. 112-124.

Baacke, Dieter. Zum Konzept und zur Operationalisierung von Medienkompetenz. 1998. URL: http://www.uni-bielefeld.de/paedagogik/agn/ag9/Texte/MKompetenz1.htm (25.10.2005).

Bader, Roland. «Legitime Partizipation. Ein Handlungsmodell der Medienpädagogik mit Computern.» Bildung in virtuellen Welten. Praxis und Theorie ausserschulischer Bildung mit Internet und Computer. Beiträge zur Medienpädagogik, Band 6. Herausgegeben von Schindler, Wolfgang; Bader, Roland; Eckmann, Bernhard. Frankfurt a. M:, Gemeinschaftswerk der Evangelischen Publizistik, Abt. Verlag, 2001. 356-376.

Bausch, Constanze; Sting, Stephan. "Rituelle Medieninszenierungen in Peergroups.» Das Soziale als Ritual. Zur performativen Bildung von Gemeinschaften. Herausgegeben von Christoph Wulf, Birgit Althans, Kathrin Audehm, Constanze Bausch. Michael Göhlich, Stephan Sting, Anja Tervooren, Monika WagnerWilli und Jörg Zirfas. Opladen: Leske + Budrich, 2001. 249-323.

Bittlingmayer, Uwe H.; Bauer, Ullrich. «Ungleichheit - Bildung - Herrschaft. Zur politischen Soziologie der Milieutheorie Michael Vesters. Milieuspezifische Bildungsungleichheit. Genese und Konsequenzen.» Soziale Milieus und Wandel der Sozialstruktur. Die gesellschaftlichen Herausforderungen und die Strategien der sozialen Gruppen. Festschrift für Michael Vester zum 65. Geburtstag. 
Herausgegeben von Helmut Bremer und Andrea Lange-Vester. Wiesbaden: VS-Verlag, 2006. 212-234

Bonfadelli, Heinz. Die Wissenskluft-Perspektive. Massenmedien und gesellschaftliche Information. Konstanz: UVK Medien, 1994.

Bonfadelli, Heinz; Bucher, Priska. "Alte und neue Medien im Leben von Jugendlichen mit Migrationshintergrund.» Grenzenlose Cyberwelt? Zum Verhältnis von digitaler Ungleichheit und neuen Blldungszugängen für Jugendliche. Herausgegeben von Kompetenzzentrum Informelle Bildung. Wiesbaden: VS-Verlag, 2007. 222-232.

Bonfadelli, Heinz; Moser, Heinz. Medien und Migration. Europa als multikultureller Raum? Wiesbaden: VS Verlag für Sozialwissenschaften, 2007.

Bremer, Helmut. Soziale Milieus, Habitus und Lernen. Zur sozialen Selektivität des Bildungswesens am Beispiel der Weiterbildung. Weinheim: Juventa, 2007.

Brüggen, Niels. «Kompetenter Medienumgang aus Sicht der Heranwachsenden.» Medienhandeln in Hauptschulmilieus. Mediale Interaktion und Produktion als Bildungsressource. Herausgegeben von Ulrike Wagner. München: Kopaed, 2008. 186-207.

Brüggen, Niels; Wagner, Ulrike. «Pädagogische Konsequenzen.» Medienhandeln in Hauptschulmilieus. Mediale Interaktion und Produktion als Bildungsressource. Herausgegeben von Ulrike Wagner. München: Kopaed, 2008. 223-246.

Eggert, Susanne; Gebel, Christa; Wagner, Ulrike. «Die vertieften Handlungsschwerpunkte Spielen, Kommunizieren und Produzieren.» Medienhandeln in Hauptschulmilieus. Mediale Interaktion und Produktion als Bildungsressource. Herausgegeben von Ulrike Wagner. München: Kopaed, 2008. 119-185.

Feierabend, Sabine; Rathgeb, Thomas. JIM-Studie 2008. Jugend, Information, (Multi-) Media. Stuttgart: Medienpädagogischer Forschungsverbund Südwest, 2008.

Gebhardt, Julian. «Inszenierung und Verortung von Identität in der computervermittelten Kommunikation. Rahmenanalytische Überlegungen am Beispiel des ¿Online-Chatı.»kommunikation@gesellschaft, Jg., 2001, Beitrag 7. 1 - 21 URL: http://www.soz.uni-frankfurt.de/K.G/B7_2001_Gebhardt.pdf (19.11.2008).

Groeben, Norbert. «Dimensionen der Medienkompetenz: Deskriptive und normative Aspekte.» Medienkompetenz. Voraussetzungen, Dimensionen, Funktionen. Herausgegeben von Norbert Groeben und Bettina Hurrelmann. Weinheim und München: Juventa, 2002. 160-200.

Henke, Ursula; Mogge-Grotjahn, Hildegard; Huster, Ernst-Ulrich. «E-exclusion oder e-inclusion?» Handbuch soziale Armut und soziale Ausgrenzung. Herausgegeben von Ernst-Ulrich Huster, Jürgen Boeckh und Hildegard Mogge-Grotjahn. Wiesbaden: VS-Verlag für Sozialwissenschaften. 470-482.

Iske, Stefan; Klein, Alex; Kutscher, Nadia; Otto, Hans-Uwe. «Virtual Inequality and Informal Education: An Empirical Analysis of Young Peoples' Internet Use and 
its Significance for Education and Social Participation.» Technology, Pedagogy and Education - special issue theme Technology, pedagogy and education glimpses of the wider pictures. Volume 17, Issue 2, 2008. 131-141.

Iske, Stefan; Klein, Alex; Kutscher, Nadia; Otto, Hans-Uwe. "Virtuelle Ungleichheit und informelle Bildung. Eine empirische Analyse der Internetnutzung Jugendlicher und ihre Bedeutung für Bildung und gesellschaftliche Teilhabe.» Grenzenlose Cyberwelt? Zum Verhältnis von digitaler Ungleichheit und neuen Blldungszugängen für Jugendliche. Herausgegeben von Kompetenzzentrum Informelle Bildung. Wiesbaden: VS-Verlag, 2007. 65-92.

Iske, Stefan; Klein, Alex; Kutscher, Nadia. Digitale Ungleichheit und formaler Bildungshintergrund - Ergebnisse einer empirischen Untersuchung über Nutzungsdifferenzen von Jugendlichen im Internet. 2004a. URL: http://www.kibbielefeld.de/externelinks2005/digitaleungleichheit.pdf (26.2.2006).

Iske, Stefan; Klein, Alex; Kutscher, Nadia. «Nutzungsdifferenzen als Indikator für soziale Ungleichheit im Internet.»kommunikation@gesellschaft, Jg. 5, Beitrag 3, 2004b. URL: http://www.soz.uni-frankfurt.de/K.G/B3_2004_Iske_Klein_Kutscher.pdf (19.2.2006).

Kiessling, Matthias. "Jugend 2.0? Der Einfluss der Bildung auf die Nutzung des Internets.» merz - Zeitschrift für Medienpädagogik, 2/2008. 21-22.

Klein, Alex. «Soziales Kapital Online»: Soziale Unterstützung im Internet. Eine Rekonstruktion virtualisierter Formen sozialer Ungleichheit. Bielefeld, 2008. URL: http://bieson.ub.uni-bielefeld.de/volltexte/2008/1260/pdf/Klein_Alexandra_ Dissertation.pdf (6.10.08).

Klein, Alex. Online-Beratung für alle? Themenfelder, Unterstützungsmuster und Reichweiten netzbasierter Beratung. 2005. URL: http://www.kib-bielefeld.de/ externelinks2005/OnlineBeratungfueralle.pdf (15.2.08).

Klein, Alex. «Von «Digital Divide〉 zu 〈Voice Divide〉. Beratungsqualität im Internet.» Informelle Bildung online. Perspektiven für Bildung, Jugendarbeit und Medienpädagogik. Herausgegeben von Hans-Uwe Otto und Nadia Kutscher. Weinheim und München: Juventa, 2004. 97-121.

Kutscher, Nadia. «Virtuelle Räume Jugendlicher - die Wirkmacht kulturellen Kapitals bei der Nutzung des Internet.» Multilokalität und Vernetzung. Beiträge zur technikbasierten Gestaltung jugendlicher Sozialräume. Herausgegeben von Claus J. Tully. Weinheim und München: Juventa, 2009. 158-174.

Kutscher, Nadia. «Beteiligung von Jugendlichen zwischen Interessen, Erwartungen und Lebensalltag.» Kinder- und Jugendbeteiligung in Deutschland - Entwicklungsstand und Handlungsansätze. Herausgegeben von Bertelsmann Stiftung. Gütersloh, 2007. 187-203.

Löw, Martina. Raumsoziologie. Frankfurt am Main: Suhrkamp, 2001.

Niesyto, Horst. Medienpädagogik und soziokulturelle Unterschiede. Eine Studie zur Förderung der aktiven Medienarbeit mit Kindern und Jugendlichen aus 
bildungsmässig und sozial benachteiligten Verhältnissen. Baden-Baden: Medienpädagogischer Forschungsverbund Südwest, 2000.

Niesyto, Horst. «Medienpädagogik, Mediensozialisation und soziale Benachteiligung.» Grenzenlose Cyberwelt? Zum Verhältnis von digitaler Ungleichheit und neuen Blldungszugängen für Jugendliche. Herausgegeben von Kompetenzzentrum Informelle Bildung. Wiesbaden: VS-Verlag, 2007. 153-174.

(N)Onliner-Atlas (2008). Herausgegeben von der Initiative D21. URL: http://www. initiatived21.de/category/nonliner-atlas (6.12.08)

Otto, Hans-Uwe; Kutscher, Nadia; Klein, Alex; Iske, Stefan. Soziale Ungleichheit im virtuellen Raum: Wie nutzen Jugendliche das Internet? Erste Ergebnisse einer empirischen Untersuchung zu Online-Nutzungsdifferenzen und Aneignungsstrukturen von Jugendlichen. 2004 (überarbeitete Version 2005). URL: http:// www.bmfsfj.de/RedaktionBMFSFJ/Abteilung5/Pdf-Anlagen/jugend-internetlangfassung, property=pdf (19.2.2006).

Pirker, Bettina. «Medien.Technik.Raum. Technikkonstruierte Räume als interkulturelle Spielplätze der Identität.» Multilokalität und Vernetzung. Beiträge zur technikbasierten Gestaltung jugendlicher Sozialräume. Herausgegeben von Claus J. Tully. Weinheim und München: Juventa, 2009. 91-100.

Schäfer, Miriam; Lojewski, Johanna. Internet und Bildungschancen - Zur sozialen Realität des virtuellen Raumes. München: Kopäd, 2007.

Schäffer, Burkhard. «Das Internet: ein Medium kultureller Legitimität in Bildungskontexten?» Zum Bildungswert des Internet. Herausgegeben von Winfried Marotzki; Dorothee Meister; Uwe Sander. Opladen: Leske + Budrich, 2000. 259 -285 .

Schell, Fred. «Aktive Medienarbeit im Zeitalter des Web 2.0.» merz - Zeitschrift für Medienpädagogik 2/2008. 9 - 12.

Schönberger, Klaus. «Internet und Netzkommunikation im sozialen Nahbereich. Anmerkungen zum langen Arm des 〈real lifes.» forum medienethik 2/2000: Netzwelten, Menschenwelten, Lebenswelten. Kommunikationskultur im Zeichen von Multimedia. 33-42.

Schorb, Bernd; Kiessling, Matthias; Würfel, Maren; Keilhauer, Jan. Medienkonvergenz Monitoring Report 2008. Jugendliche in konvergierenden Medienwelten. 2008. URL: http://www.uni-leipzig.de/ umfmed/Medienkonvergenz_Monitoring_Report08.pdf (27.8.2008).

Tillmann, Angela. Identitätsspielraum Internet. Weinheim und München: Juventa, 2008.

Tulodziecki, Gerd. Medien in Erziehung und Bildung. Grundlagen und Beispiele einer handlungs- und entwicklungsorientierten Medienpädagogik. Bad Heilbrunn: Klinkhardt Verlag, 1997.

Wagner, Ulrike (Hrsg.): Medienhandeln in Hauptschulmilieus. Mediale Interaktion und Produktion als Bildungsressource. München: Kopaed, 2008. 
Welling, Stefan. «Medienpädagogisches 〈Brötchenbacken〉 - ein integriertes Modell medienpädagogischer Praxisentwicklung.» MaC* - Reloaded: Perspektiven aus der Skepsis. Herausgegeben von Wolfgang Schindler. Chemnitz: RabenStück Verlag für Kinder- und Jugendhilfe, 2005. 209-233.

Welling, Stefan. Computerpraxis Jugendlicher und medienpädagogisches Handeln. München: kopaed, 2008.

World Vision (Hrsg.). Kinder in Deutschland 2007. Frankfurt am Main: Fischer, 2007. 